\title{
Metastasis: A Bane of Breast Cancer Therapy
}

Authors:

Chidalu A. Edechi, ${ }^{1}$ Nnamdi M. Ikeogu, ${ }^{2}$ Lucas Evangelista de Lima Terceiro, Jude E. Uzonna, ${ }^{2}{ }^{1}$ Yvonne Myal ${ }^{1,3,4}$

1. Department of Pathology, Max Rady College of Medicine, University of Manitoba, Winnipeg, Canada

2. Department of Immunology, Max Rady College of Medicine, University of Manitoba, Winnipeg, Canada

3. Department of Physiology and Pathophysiology, Max Rady College of Medicine, University of Manitoba, Winnipeg, Canada

4. Research Institute in Oncology and Hematology, CancerCare Manitoba, Winnipeg, Canada

*Correspondence to yvonne.myal@umanitoba.ca

Disclosure: $\quad$ The authors have declared no conflicts of interest.

Acknowledgements: The authors wish to acknowledge the grant support from CancerCare Manitoba Foundation. Chidalu A. Edechi and Nnamdi M. Ikeogu contributed equally to the manuscript.

Received:

19.02.20

Accepted:

31.03 .20

Keywords:

Breast cancer, chemotherapy, epithelial-mesenchymal transition (EMT), immune system, immunotherapy, metastasis.

Citation:

EMJ. 2020;5[3]:55-62.

\section{Abstract}

The underlying mechanisms of metastasis in patients with breast cancer is still poorly understood. Approximately $6 \%$ of patients with breast cancer present with metastasis at the time of diagnosis. Metastatic breast cancer is difficult to treat and patients with breast cancer with distant metastasis have a significantly lower 5-year survival rate compared to patients with localised breast cancer $(27 \%$ and 99\%, respectively). During breast cancer progression, tumour cells first metastasise to nearby draining lymph nodes and then to distant organs, primarily bone, lungs, liver, and brain. In this brief review, the authors discuss breast cancer metastasis, the role of epithelial-mesenchymal transition and the contributions of the immune system to the metastatic process. The authors also briefly discuss whether there is any relationship between tumour size and metastatic potential, and recent advances in treatment for metastatic breast cancer. The studies highlighted suggest that immunotherapy may play a more significant role in future patient care for metastatic breast cancer.

\section{INTRODUCTION}

\section{Breast Cancer: From Detection to Treatment}

Breast cancer is one of the most commonly diagnosed cancers among women.' Despite advances in treatment for breast cancer, 6-10\% of women diagnosed with breast cancer

approximately $10 \%$ of women currently fail primary management strategies for early breast cancer and go on to develop recurrence, ultimately succumbing to the disease within 5 years. For those who present with late stage breast cancer, the odds of dying within that same timeframe increases to $30 \% .^{2}$ Furthermore, 
have Stage IV, or metastatic, breast cancer that has spread to other organs, primarily the lung, liver, brain or bone; $85 \%$ of breast cancer deaths are attributable to metastasis. ${ }^{2}$ Although several subtypes of breast cancer have now been identified, breast cancers are often categorised into four major molecular subtypes: luminal A and luminal $B$, based on oestrogen receptor (ER) and progesterone receptor (PR) status; human epidermal growth factor receptor 2 (HER2)-positive; and basal-like or triple-negative breast cancers (TNBC), which are ER-, PR-, and HER2-negative. ${ }^{3}$

Detection of breast cancer can be through self-detection but, most often, breast cancers are identified by mammographic screening ${ }^{4}$ followed by breast biopsy. ${ }^{5}$ Prolactin-inducible protein, mammaglobin, and GATA-3 are used to determine whether an unknown metastatic cancer is of breast origin. ${ }^{6}$ Additionally, there are blood-based assays which detect breast cancerassociated biomarkers such as carcinoembryonic antigen (CEA). ${ }^{7}$ Treatment for breast cancer primarily comprises surgical intervention, followed by endocrine (hormonal) therapy, chemotherapy, radiation therapy, or some combination of these therapeutic approaches. ${ }^{8}$ Endocrine therapy is recommended in patients with breast cancer with hormone (oestrogen and progesterone) receptor-positive (HRpositive) tumours. ${ }^{8}$ The menopausal status of the patient plays a major role in the decision to recommend a particular therapeutic strategy. ${ }^{9}$ While tamoxifen is the drug of choice for premenopausal patients, ${ }^{9}$ aromatase inhibitors (such as letrozole) are preferred for postmenopausal patients..$^{10}$ For patients with HR-negative breast tumours, chemotherapy is the treatment of choice and is often recommended in patients with TNBC and HER2-positive breast cancer." Radiation therapy may also be used to further shrink breast tumours after surgical intervention. ${ }^{2}$

Patients with early-stage and localised breast cancer generally have a good prognosis with approximately $99 \%$ of patients surviving over a 5-year period.' However, a recent study showed that at the cessation of 5 years of endocrine therapy, the risk of breast cancer recurrence remained relatively significant even after 15 to

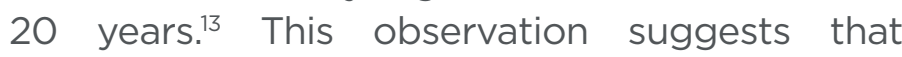
5 years of treatment is not sufficient and that a longer period of therapy should be considered to prevent breast cancer recurrence. In this review, the authors give a brief overview of the existing challenges metastatic breast cancer presents and highlight some recent advances in treatment strategies.

\section{BREAST CANCER METASTASIS: FROM PRIMARY TUMOUR TO DISTAL SITES}

Metastasis is a series of events that involve the invasion of the basement membrane at the primary tumour site, movement of the tumour cells into circulation, and subsequent dissemination to other organs. ${ }^{14}$ During metastasis, cells lose their epithelial nature and assume a mesenchymal phenotype, and extracellular matrix proteins in the basement membrane are degraded by proteolytic enzymes, such as matrix metalloproteinases.$^{14}$ Collectively, these processes allow the cancer cells to leave the primary tumour site and escape into the circulation (blood or lymphatics) in a process known as intravasation. Generally, breast cancer first spreads to lymph nodes in close proximity to the breast through the lymphatic system, while metastasis to distant organs usually occurs via the blood.15 Once in circulation, the breast cancer cells, now referred to as circulating tumour cells (CTC) ${ }_{16}^{16}$ migrate to different organs in the body. CTC are often scarce in non-metastatic breast cancer but higher in advanced breast cancer disease. ${ }^{17}$ Some markers used for CTC detection include the epithelial cell adhesion molecule, an epithelial cell marker. However, cancers can alter their expression of these markers; for instance, by undergoing epithelial-mesenchymal transition (EMT).$^{18}$ CTC are now being utilised for the early detection of breast cancer ${ }^{19}$ and the evaluation of metastatic risk in patients. ${ }^{17}$ However, there are controversies concerning the detection of CTC in the blood because of the absence of accurate and specific markers. ${ }^{18}$ Alternatively, other biomarkers, such as exosomes, may also be used for determining diagnosis or prognosis, or to predict response to therapy. ${ }^{20}$ When CTC move out of the blood to metastatic sites in a process known as extravasation, ${ }^{21}$ they are then referred to as disseminated tumour cells. ${ }^{16}$ At these metastatic sites, the disseminated tumour cells form new tumour foci and spread throughout the affected organ, ultimately contributing to 
patient mortality. It is believed that metastasis involves an interplay between cancer cells and the metastatic tissue or organ, as some cancer cells are able to better form metastasis than others and some metastatic organs are more receptive to cancer cells than others. ${ }^{22}$ The metastatic process is reviewed by Lambert et al. ${ }^{14}$

\section{Epithelial-Mesenchymal Transition}

EMT is the process by which epithelial cells acquire a mesenchymal phenotype during cancer development. ${ }^{23}$ EMT is induced by key transcription factors such as Snail, Slug, Twist, and ZEB1 ${ }^{14}$ and is characterised by loss of cell-cell adhesion, apical-basal polarity, and expression of epithelial markers such as E-cadherin and cytokeratin, while mesenchymal markers, including $\mathrm{N}$-cadherin and vimentin, are upregulated. ${ }^{24}$ This shift from an epithelial to a mesenchymal phenotype enables the cancer cells to detach from the primary tumour, invade the basement membrane, and migrate to a distant organ and establish there. ${ }^{25}$ Following transition, the cancer cells then acquire the ability to initiate tumour formation, which is a typical feature of cancer stem cells. ${ }^{26}$ This tumourinitiating ability is a critical step in the metastatic process as it ensures that the disseminated breast cancer cells are capable of forming tumours at metastatic sites. ${ }^{14}$ Across different breast cancer subtypes, TGF- $\beta$ signalling has been shown to be important for EMT and is potentially a master regulator of this process in breast cancer cells. This has been reviewed by Singh et al. ${ }^{27}$

\section{The Immune Response}

The immune system plays a critical role in the progression of many cancers including breast cancer. Similar to certain components of the immune system that are known to participate in the prevention and elimination of cancer, other components of the immune system can promote cancer progression and metastasis. ${ }^{28}$ Myeloidderived suppressor cells (MDSC), alternatively activated (M2) macrophages, and regulatory $T$ cells have been shown to secrete factors that suppress anti-tumour immune responses. ${ }^{29}$ MDSC secrete TGF- $\beta$ and vascular endothelial growth factor (VEGF) which stimulate EMT and angiogenesis, facilitating tumour growth and metastasis. ${ }^{30}$ MDSC also release the anti-inflammatory cytokine IL-10: a potent suppressor of anti-tumour immune responses. ${ }^{30}$ Additionally, macrophages in the tumour microenvironment have been shown to display the M2 phenotype; M2 macrophages are important for wound healing and tissue repair. ${ }^{31}$ Like MDSC, M2 macrophages release TGF- $\beta$, IL-10, and chemokines which promote breast cancer growth and metastasis. ${ }^{32}$ In addition to suppressing anti-tumour cytotoxic and helper $T$ cells via cell-to-cell contact, regulatory $T$ cells also secrete IL-10 which dampens anti-tumour immune responses. ${ }^{33}$ Studies also indicate that neutrophils, another group of immune cells, can enhance metastasis by inhibiting cytotoxic $T$ cell function, ${ }^{34}$ secreting leukotrienes, and promoting the proliferation of tumour-initiating cells at the metastatic environment. ${ }^{35}$

\section{Does Tumour Size Matter?}

Although previous studies suggested that the relationship between tumour size and metastasis was linear, recent data from a large population study of more than 800,000 patients with breast cancer showed that it is not. ${ }^{36}$ This study showed that at the time of diagnosis, a patient with a tumour $7 \mathrm{~cm}$ in diameter has an approximately equal chance of having lymph node metastasis compared to a patient with a $15 \mathrm{~cm}$ tumour $(71.8 \%$ versus $71.3 \%$, respectively). ${ }^{37}$ The discrepancy between previous studies and the current study may be attributed to the small sample size and inaccurate methods of those previous studies. In addition to axillary lymph node metastasis, studies also show that larger tumour sizes correlated with worse clinical outcomes. ${ }^{38}$ However, many patients with TNBC, especially the subset that express basal markers such as cytokeratin, do not follow this trend. ${ }^{36}$ Indeed, smaller basal tumours have been associated with more lymph node metastasis than non-basal tumours and had worse disease outcomes than expected; larger basal tumours were associated with better outcomes than expected. ${ }^{39}$ Most of the mutations found in the primary tumour of a patient with basal-like breast cancer were also found in metastatic cells of the patient, suggesting that the primary tumour cells already had the necessary features needed to metastasise. ${ }^{40}$ Other parameters such as biomarker expression, e.g., Ki67, were found to correlate with both lymph node metastasis and 
tumour size. ${ }^{41}$ It appears that size is not the only determining factor for metastatic spread; the subtype and intrinsic biology of the tumour may play critical roles as well.

\section{PREFERRED SITES FOR BREAST CANCER METASTASIS}

The draining, or sentinel, lymph node in the axilla, which is located in close proximity to the breast tumour site, is usually the first site of metastasis. ${ }^{42}$ Breast cancer then spreads to distal sites, most frequently bone, lungs, liver, and the brain. ${ }^{43}$ Estimates from a recent study showed that at the time of diagnosis, patients with metastatic breast cancer were found to most frequently present with bone metastasis (3.28\%), followed by lung (1.52\%) and liver (1.20\%) metastasis, while the brain was the least common site for metastasis (0.35\%). ${ }^{44}$

\section{Metastasis to Bone}

Current understanding suggests that metastatic breast cancer cells are able to take advantage of the natural remodelling process in the bone to facilitate metastasis by disrupting the balance between formation and resorption of bone, leading to bone loss (osteolysis) or abnormal bone formation. ${ }^{45}$ One process for causing this disruption is by secreting factors to modulate their environment. ${ }^{46}$ Breast cancer cells have been reported to secrete IL-11 and matrix metalloproteinases which can stimulate osteoclasts to produce growth factors that, in turn, promote the growth and survival of the metastatic breast cancer cells in the bone. ${ }^{46}$ Interestingly, results from recent studies also suggest that prolactin overexpression in breast tumours can shorten the time to development of bone metastasis and contribute to osteolysis induced by the metastatic breast cancer cells. ${ }^{47}$ Among all breast cancer subtypes, patients with luminal breast cancer have the highest rate of bone metastasis. ${ }^{48}$

\section{Metastasis to the Lungs}

Breast cancer cells released from the primary tumour may also spread to the lungs. ${ }^{49}$ Lung metastasis is usually observed 5 years after initial breast cancer diagnosis, with $>60 \%$ mortality rate following lung metastasis. ${ }^{49}$ The subtype of breast cancer affects the frequency of lung metastasis. Patients with TNBC were reported to be more likely to develop lung metastasis compared to other subtypes. ${ }^{48}$ The development of lung metastasis in patients with breast cancer may be because of an inherent ability of breast cancer cells to the lungs, or an ability to interact with the lung microenvironment. Premalignant breast cells have been shown to invade the lungs after injection into the blood, suggesting that breast cancer cells may have some intrinsic ability to establish in the lungs. ${ }^{50}$ It has been shown in vitro that the migration and proliferation of breast cancer cells were stimulated by the conditioned medium of the lungs, suggesting that factors from the lungs promote lung metastasis. One such factor identified was selectin, a cell adhesion molecule. ${ }^{51}$

\section{Metastasis to the Liver}

Another common site for breast cancer metastasis is the liver. Liver metastasis leads to impairment of liver function with serious outcomes, and without treatment intervention life expectancy is $<8$ months. ${ }^{52}$ Patients with HER2-positive breast cancers show the highest incidence of liver metastasis. ${ }^{48}$

\section{Metastasis to the Brain}

Although less common, breast cancer can also metastasise to the brain. Patients with breast cancer and brain metastasis have poor prognosis, display neurological defects, and do not respond well to therapy. ${ }^{53}$ Due to more successful treatment of the primary tumour, patients with breast cancer live longer, and brain metastasis more frequently becomes evident later in the disease process. ${ }^{54}$ The propensity of breast cancer to metastasise to the brain seems to depend on the breast cancer subtype..$^{55}$ Brain metastasis occurs in approximately $50 \%$ of patients with TNBC, and $33 \%$ and $14 \%$ of patients with HER2-positive and HR-positive breast cancer, respectively.48

\section{ADVANCES IN TREATMENT MODALITIES FOR METASTATIC BREAST CANCER}

\section{Standard Therapeutic Drugs}

The treatment strategies utilised for patients with metastatic breast cancer depend on several 
factors. These factors are often based on two considerations: the breast cancer subtype and the stage of the disease. Generally, patients with HR-positive breast cancer are treated with endocrine therapy, patients with HER2positive breast cancer are treated with antiHER2 antibody therapies, such as trastuzumab, while triple-negative tumours are typically treated with chemotherapy. ${ }^{3}$ The initial treatment regimen for patients with metastatic HR-positive breast cancer includes endocrine therapy in combination with a cyclin-dependent kinase (CDK) 4/6 inhibitor, such as abemaciclib. Patients with resistance to endocrine therapy are treated with chemotherapy. ${ }^{3}$ For patients with HER2positive breast cancer, the first line of treatment includes a combination of an anti-HER2 antibody therapy, such as trastuzumab, and a chemotherapy, e.g., a taxane. If this initial treatment is unsuccessful, trastuzumab emtansine, an antibody-drug conjugate, is then administered. ${ }^{3}$ If this treatment also fails, continuous use of the HER2-targeted therapy may be recommended, in combination with a different chemotherapeutic agent. Unlike HRand HER2-positive breast cancers, TNBC are only treated with chemotherapy because of a lack of established molecular targets, as reviewed by Waks et al. $^{3}$

\section{New Therapeutic Drugs}

Several new therapeutic agents have been recently approved by the U.S. Food and Drug Administration (FDA), while a number are still undergoing clinical trials. Sacituzumab govitecan is an antibody-drug conjugate, currently undergoing testing in clinical trials, which has shown positive effects in previously treated patients with advanced TNBC. ${ }^{56}$ Another promising drug is atezolizumab, an inhibitor of programmed death-ligand 1 , and nanoparticle albumin-bound-paclitaxel, which was shown to enhance progression-free survival in patients with breast cancer. ${ }^{57}$ To treat patients with metastatic breast tumours harbouring the BRCA1/2 mutations, poly(ADP-ribose) polymerase (PARP) inhibitors, such as olaparib, were recently approved by the FDA. ${ }^{58}$ PARP inhibitors are used to counteract the DNA repair brought about by PARP, to enhance the sensitivity of cancer cells to DNA-damaging anti-cancer therapies, and ultimately lead to cell death. ${ }^{59}$ However, many of these drugs are only successful in extending the patients' lives for a short period of time. Response rates are often poor, and many patients develop serious side effects. A study reported that among histological subtypes, patients with invasive lobular carcinoma had increased progression-free survival compared to patients with invasive ductal carcinoma; however, there was no difference in overall survival. ${ }^{60}$ Across molecular subtypes, patients with HR-positive/ HER2-positive breast tumours had better survival outcomes, while those with TNBC had worse outcomes. ${ }^{61}$ Another challenge is that following systemic therapy, a population of dormant breast cancer cells may persist and subsequently lead to relapse. ${ }^{14}$ This situation is further complicated by the cytotoxic treatments used against breast cancer mainly targeting highly proliferating cells with minimal effects on dormant cells. ${ }^{14}$

\section{Immunotherapy: Homing in on our Immune Response}

Immunotherapy is a treatment strategy that harnesses the ability of the immune system to keep diseases, including cancers, at bay. In recent years, there have been some successes in using immunotherapy to treat certain type of cancers such as melanoma, which is considered to be an immunogenic cancer, capable of eliciting an immune response. ${ }^{62}$ Breast cancer was previously not considered to be an immunogenic cancer, but it has now been shown that the presence of immune cells in some breast cancers is of prognostic and therapeutic significance. ${ }^{62}$ Breast cancers with greater influx of tumour-infiltrating lymphocytes tend to respond better to neoadjuvant chemotherapy compared to those with fewer tumourinfiltrating lymphocytes. ${ }^{29}$

Recently, some immunotherapeutic approaches have been assessed for the treatment of a limited number of patients with breast cancer. These include the use of tumour antigen-specific antibodies and adoptive transfer of activated immune cells, such as natural killer cells, dendritic cells, and T cells. ${ }^{28} \mathrm{~A}$ recent study was the first to demonstrate no signs of cancer in a patient with metastatic breast cancer following treatment by adoptive transfer of activated patient-derived tumour-specific T cells. ${ }^{63}$ Pembrolizumab, an anti- 
PD1 antibody, has been shown to be effective for patients with metastatic TNBC in clinical trials. ${ }^{64}$ Vaccines are also being explored as another treatment strategy. The use of recombinant HER2 protein in patients with advanced HER2-positive breast cancer was shown to induce an antitumour immune response and benefit a limited number of patients. ${ }^{65}$ The use of chimeric antigen receptor $\mathrm{T}$ cells is another immunotherapeutic strategy currently being developed for treatment of breast cancer. Studies using chimeric antigen receptor $T$ cells engineered to target the mucin 1 protein expressed on breast cancer cells have shown promising preclinical results ${ }^{66}$ and this therapy is currently undergoing clinical trials. ${ }^{67}$ Major challenges with these approaches include the cost of treatment and the difficulty in isolating sufficient activated tumour-specific $T$ cells from patients. However, efforts are being made to reduce these challenges through the utility of immune cells from unrelated donors without tissue rejection. ${ }^{68}$ Some current therapeutic approaches are summarised in Table 1.

\section{CONCLUSION}

The wide degree of heterogeneity that exists among breast cancers presents many challenges for both clinicians and patients. There are still significant gaps in knowledge about the metastatic process during breast cancer progression, including: why different breast cancer subtypes preferentially metastasise to specific organs; why tumour size does not always matter; and why some breast cancers trigger an immune response while others do not. Advances in the understanding of the interaction between several cancers and the immune system could lead to the use of immunotherapy as a viable option for the treatment of some patients with breast cancer with metastatic disease. There is also a need for greater understanding of key signalling pathways. However, a multidisciplinary approach to research is warranted to address these gaps in knowledge and to develop effective strategies to benefit a large number of patients. Furthermore, current comprehensive guidelines for the treatment of metastatic breast cancer provided by key European and American societies, such as the European Society for Medical Oncology (ESMO) ${ }^{69}$ and the National Comprehensive Cancer Network (NCCN), ${ }^{70}$ are largely similar in their recommendations. However, some discrepancies do exist, highlighting that further research is warranted to fully determine which therapies are most effective and beneficial for patients in the long term.

Table 1: Examples of current therapeutic approaches for metastatic breast cancer patients.

\begin{tabular}{|c|c|c|}
\hline Treatment & Breast cancer subtype & Overall survival \\
\hline \multicolumn{3}{|l|}{ Standard therapies } \\
\hline $\begin{array}{l}\text { Endocrine therapy only, or in combination with CDK 4/6 } \\
\text { inhibitor (abemaciclib) } \\
\text { Endocrine therapy and chemotherapy (taxane) }\end{array}$ & HR-positive breast cancer & 4-5 years y $^{3}$ \\
\hline $\begin{array}{l}\text { Anti-HER2 antibody (trastuzumab) and taxane } \\
\text { Trastuzumab emtansine }\end{array}$ & HER2-positive breast cancer & 5 years $^{3}$ \\
\hline Chemotherapy (taxane, anthracycline) & TNBC & 10-13 months ${ }^{3}$ \\
\hline \multicolumn{3}{|l|}{ New therapies } \\
\hline Sacituzumab govitecan & TNBC & 13 months $^{56}$ \\
\hline Atezolizumab and nanoparticle albumin-bound paclitaxel & TNBC & $\begin{array}{l}25 \text { months (patients with PD- } \\
\text { L1-positive tumours) }\end{array}$ \\
\hline Pembrolizumab & TNBC & 9 months $^{61}$ \\
\hline
\end{tabular}

CDK: cyclin-dependent kinase; HER2: human epidermal growth factor receptor 2; HR: hormone receptor; PD-L1: programmed death-ligand 1; TNBC: triple-negative breast cancer. 


\section{References}

1. Siegel $\mathrm{R}$ et al. Cancer statistics. CA Cancer J Clin. 2019;69:7-34.

2. American Cancer Society. Cancer Facts \& Figures 2019. 2019. Available at: https://www.cancer.org/ research/cancer-facts-statistics/ all-cancer-facts-figures/cancer-factsfigures-2019.html. Last accessed: 1 April 2020

3. Waks AG, Winer EP. Breast cancer treatment: a review. JAMA. 2019;321(3):288-300.

4. Kerlikowske $\mathrm{K}$ et al. Comparative effectiveness of digital versus filmscreen mammography in community practice in the United States: a cohort study. Ann Intern Med. 2011;155(8):493-502.

5. Palmer ML, Tsangaris TN. Breast biopsy in women 30 years old or less. Am J Surg. 1993;165(6):708-12.

6. Yan Z et al. Diagnostic utility of mammaglobin and GCDFP-15 in the identification of metastatic breast carcinoma in fluid specimens. Diagn. Cytopathol. 2009;37(7):475-8.

7. Lacroix M. Significance, detection and markers of disseminated breast cancer cells. Endocr Relat Cancer. 2006;13(4):1033-67.

8. Dhankhar $\mathrm{R}$ et al. Advances in novel drug delivery strategies for breast cancer therapy. Artif Cells Blood Substit Immobil Biotechnol. 2010;38(5):230-49.

9. Hirsimäki $P$ et al. Toxicity of antioestrogens. Breast J. 2002;8(2):92-6.

10. Davies $C$ et al. Long-term effects of continuing adjuvant tamoxifen to 10 years versus stopping at 5 years after diagnosis of ooestrogen receptor-positive breast cancer: ATLAS, a randomised trial. Lancet. 2013;381(9869):805-16.

11. Shah AN, Gradishar WJ. Adjuvant anthracyclines in breast cancer: what is their role? The Oncologist. 2018;23(10):1153-61.

12. Akram M, Siddiqui SA. Breast cancer management: past, present and evolving. Indian J Cancer. 2012;49(3):277-82.

13. Pan $\mathrm{H}$ et al. 20-year risks of breastcancer recurrence after stopping endocrine therapy at 5 years. $N$ Engl J Med. 2017;377:1836-46.

14. Lambert AW et al. Emerging biological principles of metastasis. Cell. 2017;168(4):670-91.

15. Chiang SPH et al. Tumor cell intravasation. Am J Physiol Cell Physiol. 2016;311:C1-14.

16. Gómez-Cuadrado L et al. Mouse models of metastasis: progress and prospects. Dis Model Mech. 2017;10:1061-74.

17. Thery $L$ et al. Circulating tumor cells in early breast cancer. JNCI Cancer Spectr. 2019;3(2):pkzO26.

18. Mamdouhi T et al. Fugitives on the run: circulating tumor cells (CTCs) in metastatic diseases. Cancer Metastasis Rev. 2019;38:297-305.

19. Nimgaonkar A et al. A novel circulating tumor cell blood test for early detection of colorectal, prostate, and breast cancers: results from 709 samples. J Clin Oncol. 2018;36:e13549.

20. Meng $Y$ et al. Exosomes: a promising avenue for the diagnosis of breast cancer. Technol Cancer Res Treat. 2019:18:1533033818821421.

21. Reymond $\mathrm{N}$ et al. Crossing the endothelial barrier during metastasis. Nat Rev Cancer. 2013;13:858-70.

22. Fidler IJ, Poste G. The 'seed and soil' hypothesis revisited. Lancet Oncol. 2008;9(8):808.

23. Kalluri R, Weinberg RA. The basics of epithelial-mesenchymal transition. J Clin Invest. 2009;119: 1420-8.

24. Zeisberg M, Neilson EG. Biomarkers for epithelial-mesenchymal transitions. J Clin Invest. 2009;119:1429-37.

25. Felipe Lima $\mathrm{J}$ et al. EMT in breast carcinoma - a review. J Clin Med. 2016;5(7):65.

26. Mani SA et al. The epithelialmesenchymal transition generates cells with properties of stem cells. Cell. 2008;133(4):704-15.

27. Singh S, Chakrabarti R. Consequences of EMT-driven changes in the immune microenvironment of breast cancer and therapeutic response of cancer cells. J Clin Med. 2019;8(5):642.

28. Edechi CA et al. Regulation of immunity in breast cancer. Cancers. 2019;11(8):1080.

29. Emens LA. Breast cancer immunobiology driving immunotherapy: vaccines and immune checkpoint blockade. Expert Rev Anticancer Ther. 2012;12(12):1597611.

30. Ma X et al. Myeloid-derived suppressor cells promote metastasis in breast cancer after the stress of operative removal of the primary cancer. Front Oncol. 2019;9:855.

31. Weisser SB et al. Generation and characterization of murine alternatively activated macrophages. Methods Mol Biol. 2013;946:225-39.

32. Tariq $\mathrm{M}$ et al. Macrophage polarization: anti-cancer strategies to target tumor-associated macrophage in breast cancer. J Cell Biochem. 2017;118(9):2484-501.

33. Watanabe MAE et al. Regulatory $T$ cells and breast cancer: implications for immunopathogenesis. Cancer Metastasis Rev. 2010;29:569-79.
34. Coffelt SB et al. IL-17-producing y $\delta$ $T$ cells and neutrophils conspire to promote breast cancer metastasis. Nature. 2015;522:345-8.

35. Wculek SK, Malanchi I. Neutrophils support lung colonization of metastasis-initiating breast cancer cells. Nature. 2015;528:413-7.

36. Foulkes WD et al. Tumor size is an unreliable predictor of prognosis in basal-like breast cancers and does not correlate closely with lymph node status. Breast Cancer Res Treat. 2009;117:199-204.

37. Sopik V, Narod SA. The relationship between tumour size, nodal status and distant metastases: on the origins of breast cancer. Breast Cancer Res Treat. 2018;170:647-56.

38. Carter CL et al. Relation of tumor size, Iymph node status, and survival in 24,740 breast cancer cases. Cancer. 1989;63:181-7.

39. O'Brien KM et al. Intrinsic breast tumor subtypes, race, and long-term survival in the Carolina Breast Cancer Study. Clin Cancer Res. 2010;16:6100-10.

40. Ding $L$ et al. Genome remodelling in a basal-like breast cancer metastasis and xenograft. Nature. 2010;464:999-1005.

41. Yuan P et al. Ki-67 expression in lumina type breast cancer and its association with the clinicopathology of the cancer. Oncol Lett. 2016;11:2101-5.

42. Rizwan A et al. Metastatic breast cancer cells in lymph nodes increase nodal collagen density. Sci Rep. 2015;5:10002

43. Wu $Q$ et al. Breast cancer subtypes predict the preferential site of distant metastases: a SEER based study. Oncotarget. 2017;8:27990-6.

44. Xiao $W$ et al. Breast cancer subtypes and the risk of distant metastasis at initial diagnosis: a populationbased study. Cancer Manag Res. 2018;10:5329-38

45. Brook $\mathrm{N}$ et al. Breast cancer bone metastases: pathogenesis and therapeutic targets. Int J Biochem Cell Biol. 2018;96:63-78.

46. Weilbaecher $\mathrm{KN}$ et al. Cancer to bone: a fatal attraction. Nat Rev Cancer. 2011;11:411-25

47. Shemanko CS. Prolactin receptor in breast cancer: marker for metastatic risk. J Mol Endocrinol. 2016;57(4):R153-65.

48. Gong $Y$ et al. Impact of molecular subtypes on metastatic breast cancer patients: a SEER population-based study. Sci Rep. 2017;7:45411.

49. Medeiros B, Allan AL. Molecular mechanisms of breast cancer metastasis to the lung: clinical and experimental perspectives. Int J Mol Sci. 2019;20(9):2272 
50. Podsypanina K et al. Seeding and propagation of untransformed mouse mammary cells in the lung. Science. 2008;321(5897):1841-4.

51. Chu JE et al. Lung-derived factors mediate breast cancer cell migration through CD44 receptor-ligand interactions in a novel ex vivo system for analysis of organ-specific soluble proteins. Neoplasia. 2014;16(2):180-91.

52. Bale $\mathrm{R}$ et al. Local treatment of breast cancer liver metastasis. Cancers. 2019;11(9):1341.

53. Niikura $\mathrm{N}$ et al. Brain metastases in breast cancer. Jpn J Clin Oncol. 2014;44(12):1133-40.

54. Franchino $\mathrm{F}$ et al. Mechanisms and therapy for cancer metastasis to the brain. Front Oncol. 2018;8:161.

55. Brosnan EM, Anders CK. Understanding patterns of brain metastasis in breast cancer and designing rational therapeutic strategies. Ann Transl Med. 2018;6(9).

56. Bardia A et al. Sacituzumab govitecan-hziy in refractory metastatic triple-negative breast cancer. N Engl J Med. 2019;380:741-51.

57. Schmid $P$ et al. Atezolizumab and nab-paclitaxel in advanced triplenegative breast cancer. N Engl J Med. 2018:379:2108-21.
58. Robson $\mathrm{M}$ et al. Olaparib for metastatic breast cancer in patients with a germline BRCA mutation. $\mathrm{N}$ Engl J Med. 2017;377:523-33.

59. McCann KE, Hurvitz SA. Advances in the use of PARP inhibitor therapy for breast cancer. Drugs Context. 2018;7:212540.

60. Mathew A et al. Distinct pattern of metastases in patients with invasive lobular carcinoma of the breast. Geburtshilfe Frauenheilkd. 2017;77(6):660-6.

61. Chen S et al. Prognostic factors and survival outcomes according to tumor subtype in patients with breast cancer lung metastases. PeerJ. 2019;7:e8298.

62. Denkert $C$. The immunogenicity of breast cancer-molecular subtypes matter. Ann Oncol. 2014;25:1453-5.

63. Zacharakis $\mathrm{N}$ et al. Immune recognition of somatic mutations leading to complete durable regression in metastatic breast cancer. Nat Med. 2018;24:724-30.

64. Adams $\mathrm{S}$ et al. Pembrolizumab monotherapy for previously treated metastatic triple-negative breast cancer: cohort A of the Phase II KEYNOTE-086 study. Ann Oncol Off J Eur Soc Med Oncol. 2019:30: 397-404.
65. Curigliano $\mathrm{G}$ et al. A Phase I/ II trial of the safety and clinical activity of a HER2-protein based immunotherapeutic for treating women with HER2-positive metastatic breast cancer. Breast Cancer Res Treat. 2016;156:301-10.

66. Zhou $R$ et al. CAR T cells targeting the tumor MUC1 glycoprotein reduce triple-negative breast cancer growth. Front Immunol. 2019;10:1149.

67. Minerva Biotechnologies Corporation. Autologous huMNC2-CAR44 T cells for breast cancer targeting cleaved form of $\mathrm{MUCl}\left(\mathrm{MUCl}^{*}\right)$. NCTO4020575. https://clinicaltrials. gov/ct2/show/NCTO4020575.

68. Strønen E et al. Targeting of cancer neoantigens with donor-derived $T$ cell receptor repertoires. Science. 2016;352(6291):1337-41.

69. Cardoso $\mathrm{F}$ et al. 4th ESO-ESMO international consensus guidelines for advanced breast cancer (ABC 4) Ann Oncol. 2018;29(8):1634-57.

70. The National Comprehensive Cancer Network (NCCN) Guidelines for Patients. Breast Cancer: Metastatic. 2018. Available at: nccnquickguidebreast_metastatic-patient.pdf. Last accessed: 1 April 2020. 